\title{
Treatment of Early Hip Osteoarthritis: Ultrasound-Guided Platelet Rich Plasma versus Hyaluronic Acid Injections in a Randomized Clinical Trial
}

\author{
Carlo Doria ${ }^{1}$ Giulia Raffaella Mosele ${ }^{1}$ Gianfilippo Caggiari ${ }^{1} \quad$ Leonardo Puddu $^{1}$ Emanuele Ciurlia ${ }^{1}$ \\ ${ }^{1}$ Department of Orthopedics, University of Sassari, Sassari, Italy \\ Joints 2017;5:152-155. \\ Address for correspondence Giulia Raffaella Mosele, MD, \\ Department of Orthopedics, Azienda Ospedaliera Univeristaria of \\ Sassari, Viale San Pietro 43/B, Sassari, Italy \\ (e-mail: moselegr@gmail.com).
}

\begin{abstract}
Purpose The aim of this study was to compare the clinical efficacy of ultrasoundguided intra-articular injections of autologous platelet rich plasma (PRP) versus hyaluronic acid (HA) for symptomatic early osteoarthritis (OA) of the hip.

Methods A prospective controlled double-blinded randomized trial on 80 patients with hip OA was conducted. The patients were divided in two groups of 40 patients each: group 1 underwent three PRP intra-articular ultrasound-guided injections, whereas group 2 underwent three HA injections. WOMAC, VAS, and Harris Hip Score were evaluated for both groups before and at 6 and 12 months after treatment.

Keywords

- hip

- osteoarthritis

- hyaluronic acid

- platelet rich plasma

- ultrasound

- injection Results The two groups were comparable in age, sex, body mass index, and severity of hip OA. Both groups showed a significant improvement from baseline at 6-month and 12-month follow-ups for all the outcome measures. No major complications were observed during the treatment and at follow-ups in both the groups.

Conclusion PRP did not offer significantly better results compared with HA in patients with moderate signs of $\mathrm{OA}$, and thus it should not be considered as first-line treatment. Level of Evidence Level II, randomized controlled trial.
\end{abstract}

\section{Introduction}

Osteoarthritis (OA) is a common, painful condition that affects adults and causes disability in the United States and Europe. Unfortunately, no agents are available to halt OA progression. Analgesics and nonsteroidal anti-inflammatory drugs (NSAIDs) have suboptimal effectiveness, and there is a concern of systemic side effects. A wide challenge is the development of appropriate and effective therapy in patients with OA. Currently, the most suitable route for administering OA therapy appears to be intra-articular injections that allow accumulation of critical doses of the drug within the damaged area and also reduce the risk of systemic side effects. ${ }^{1}$ In the last decade, hyaluronic acid (HA), and more recently, platelet rich plasma (PRP) injections have been used in the treatment of hip osteoarthritis $(\mathrm{OA}) .^{2-9}$
With progressive aging of the population, the number of patients with hip OA will increase. The aim of our study was to compare the efficacy of the two most debated treatments for early hip OA: HA and PRP. Our hypothesis is that differences in clinical outcomes between HA and PRP are not significant.

\section{Methods}

This study was designed as a prospective double-blinded, randomized controlled trial on patients with hip OA. The study was approved by the local ethics committee of the University of Sassari.

\section{Participants}

All patients provided their consent for using their data. Patients who were confirmed eligible and agreed to enter
DOI https://doi.org/ $10.1055 / \mathrm{s}-0037-1605584$. ISSN 2282-4324.
Copyright @ 2017 Georg Thieme Verlag KG Stuttgart · New York
License terms

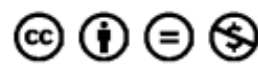


the study were randomized and treated with either three intra-articular PRP injections or three intra-articular HA injections. Inclusion criteria were symptomatic early $\mathrm{OA}$ of the hip (Kellgren-Lawrence: grade 0-2) documented by $\mathrm{X}$-ray taken within the past 6 months and age range between 40 and 72 years. Exclusion criteria were previous hip surgery, intra-articular treatments with steroids within 3 months prior to enrollment in this study, treatments with NSAIDs within 15 days prior to enrollment, chronic use of NSAIDs (defined as taking NSAIDs regularly every week for the past 6 months), steroids or chemotherapy drugs, inflammatory arthritic conditions (e.g., rheumatoid arthritis), and major comorbidities, such as poorly controlled diabetes, cardiac heart failure, chronic obstructive pulmonary disease, untreated depression, and blood disorders (thrombophilia, thrombocytopenia, anemia with hemoglobin $<9 \mathrm{~g} / \mathrm{dL}$ ). Those patients with a positive history of blood disorders had a cell blood count (CBC) 1 week prior to inclusion in the study. Because this study utilized an injection technique that may be inaccurate in obese subjects, patients with a BMI over 30 were also excluded.

The subjects enrolled were comparable in age, sex, BMI, and diagnosis of hip OA. Both groups had 12 months of follow-up. There was no drop out. In all patients, a 5 to $10 \mathrm{MHz}$ multifrequency linear probe aligned with the long axis of the femoral head was used.

\section{Interventions}

Patients were divided into two groups of 40 patients each.

Patients randomized to group 1 (PRP) received three weekly intra-articular injections of autologous PRP. The procedure of PRP preparation consisted of a $150-\mathrm{mL}$ venous blood sample. Then, two centrifugations (first at 1,480 rpm for 6 minutes to separate erythrocytes and second at $3,400 \mathrm{rpm}$ for 15 minutes to concentrate platelets) produced a unit $(20 \mathrm{~mL})$ of PRP. The unit of PRP was divided into four small units of $5 \mathrm{~mL}$ each. One unit was sent to the laboratory for analysis of platelet concentration and for a quality test, whereas three units were stored at $-30^{\circ} \mathrm{C}$.

Patients randomized to group 2 (HA) received three weekly intra-articular injections of hyaluronic acid (Hyalubrix 15 mg/mL, Fidia; Abano Terme, Italy).

Ultrasound-guided intra-articular injections of the hip were performed by the insertion of a 20- to 22-gauge spinal needle (90-120 mm) under sterile conditions. ${ }^{9}$ PRP and HA were injected at the base of the femoral neck, and a complete evacuation of intra-articular fluid, if present, was performed. The anterosuperior, parasagittal approach allows for an injection over the femoral head, and the PRP/HA is evenly distributed on the cartilage of both the femoral head and the acetabulum. Proper needle position was confirmed by direct visualization of the liquid PRP/HA that was injected. ${ }^{10}$

All the study subjects were blinded to treatment. The principal investigator (C.D.) was unblinded to treatment and was only involved in the initial assessment of the patient and actual injections. All the baseline and follow-up visits were performed by an examiner who was blinded to the treatment throughout the study.

\section{Outcomes Measures}

Both groups underwent 6- and 12-month follow-ups consisting of clinical exam and subjective and functional assessment. WOMAC score, ${ }^{11}$ Harris Hip Score, ${ }^{12}$ and pain VAS were assessed at the baseline evaluation and at 6 and 12 months after the last injection.

The primary outcome was a reduction in pain intensity as measured by the WOMAC subscale, ${ }^{6}$ which evaluates three items (pain, stiffness, and disability) for a total of 24 points (Likert format, 0-4), and by the $100-\mathrm{mm}$ VAS for pain intensity (lower scores are associated with less pain). The domain of pain in the traditional Harris hip score, ${ }^{7}$ in which higher scores were associated with less pain (out of 44 points), was used to further assess pain relief.

Secondary efficacy outcomes also included changes in physical function according to the WOMAC subscale (17 items for a total of 68 points) and the Harris hip score ( 7 items for a total of 47 points).

Adverse events during and after treatments in the two groups were also recorded and compared.

\section{Data Analysis}

All data were expressed as means and standard deviations. Statistical analysis was performed by using the SPSS software version 15.0 (SPSS Inc., Chicago, United States).

The Mann-Whitney U-test was used to compare the two groups for all the outcome variables. A $p$-value of $<0.05$ was considered significant.

\section{Results}

No significant differences were observed between the groups for baseline characteristics (-Table $\mathbf{1}$ ).

There was no drop out at the follow-up visits. Follow-up evaluation revealed a statistically significant improvement of all clinical scores from baseline evaluation to 6- and 12-month follow-ups in both the treatment groups (-Table 2).

There was a significant reduction from baseline in the WOMAC pain scores at 6 -month follow-up ( $p=0.00047$ for group $1, p=0.00063$ for group 2 ) and at 12-month follow-up ( $p=0.00607$ for group $1, p=0.00591$ for group 2$)$. The results were confirmed by reductions in the VAS scale (6-month follow-up: $p=0.00062$ for group $1, p=0.00070$ for group 2 ; 12 -month follow-up: $p=0.00606$ for group $1, p=0.00654$ for group 2) and the Harris pain subscale (6-month follow-up:

Table 1 Baseline characteristics of study population (means \pm SDs)

\begin{tabular}{|l|l|l|l|}
\hline $\begin{array}{l}\text { Baseline } \\
\text { characteristics }\end{array}$ & $\begin{array}{l}\text { Group 1 } \\
\text { (PRP) }\end{array}$ & $\begin{array}{l}\text { Group 2 } \\
\text { (HA) }\end{array}$ & -Value \\
\hline Age & $67.3 \pm 5.8$ & $68 \pm 4.6$ & ns \\
\hline BMI & $24.3 \pm 3.8$ & $25 \pm 3.8$ & ns \\
\hline OA severity & $1.5 \pm 0.5$ & $1.5 \pm 0.5$ & ns \\
\hline
\end{tabular}

Abbreviations: HA, hyaluronic acid; ns, nonsignificant; PRP, platelet rich plasma. 
Table 2 Comparison between groups for outcome scores (means \pm SDs)

\begin{tabular}{|l|l|l|l|l|l|l|}
\hline \multicolumn{3}{|l|}{} & \multicolumn{2}{l|}{ Group 1 (PRP) } & \multicolumn{3}{l|}{ Group 2 (HA) } \\
\hline Outcome measure & Baseline & $6 \mathrm{mo}$ & $12 \mathrm{mo}$ & Baseline & 6 mo & $12 \mathrm{mo}$ \\
\hline WOMAC-pain & $23.7 \pm 2.1$ & $7.8 \pm 3.8$ & $7.4 \pm 2.5$ & $24 \pm 1.9$ & $9.7 \pm 4.5$ & $9 \pm 5.6$ \\
\hline WOMAC-stiffness & $3.8 \pm 4.1$ & $2.1 \pm 3.6$ & $2 \pm 4.2$ & $4.3 \pm 5.3$ & $3.1 \pm 3.2$ & $3.1 \pm 4.3$ \\
\hline WOMAC-function & $29.4 \pm 2.6$ & $12.3 \pm 3.6$ & $12 \pm 3.8$ & $28.5 \pm 2.5$ & $11.3 \pm 4.5$ & $10.9 \pm 4.2$ \\
\hline VAS & $7.5 \pm 2.1$ & $6.3 \pm 3.3$ & $6.4 \pm 2.9$ & $7.8 \pm 1.9$ & $6.3 \pm 2.9$ & $6.1 \pm 2.3$ \\
\hline Harris Hip score & $64 \pm 10.3$ & $75 \pm 11.5$ & $78 \pm 11.3$ & $62 \pm 9.8$ & $74 \pm 12.3$ & $75 \pm 11.4$ \\
\hline
\end{tabular}

Abbreviations: HA, hyaluronic acid; PRP, platelet rich plasma; WOMAC, Western Ontario and McMaster Universities Osteoarthritis Index.

$p=0.0004$ for group $1, p=0.0006$ for group $2 ; 12$-month follow-up: $p=0.0021$ for group $1, p=0.0033$ for group 2 ).

Disability was significantly reduced from baseline in the self-reported WOMAC subscale at the 6-month follow-up ( $p=0.0142$ for group $1, p=0.0158$ for group 2 ) and at the 12-month follow-up ( $p=0.0306$ for group $1, p=0.0402$ for group 2 ) time points. Function was significantly ameliorated also at the Harris hip score at 6 months ( $p=0.0005$ for group $1, p=0.0003$ for group 2 ) and at 12 months ( $p=0.0031$ for group $1, p=0.0037$ for group 2 ).

On comparing the two treatments for adverse events, a significantly higher postinjective pain reaction was observed in group 1 ( $p=0.043$ ). However, this reaction was self-limiting within few weeks without compromising the long-term outcomes. No major complications were reported, neither at 6- or 12-month follow-up.

\section{Discussion}

Hyaluronic acid (HA) produced by synoviocytes, fibroblasts, and chondrocytes, is the major chemical component of synovial fluid. It is essential for the viscoelastic properties of the fluid because of high viscosity and has a protective effect on articular cartilage and soft tissue surfaces of joints. In OA, the concentration and the molecular weight of HA are reduced, resulting in synovial fluid with lower elasticity and viscosity, including dilutional effects, reduced hyaluronan synthesis, and free-radical degradation, which are the factors that contribute to the lower concentrations of HA. When the viscoelasticity of synovial fluid is reduced, the transmission of mechanical force to cartilage may increase its susceptibility to mechanical damage. Therefore, the restoration of normal articular homoeostasis is the rationale for HA injections into osteoarthritic joints. Besides viscosupplementation, HA is a provider of other positive biological properties, such as antiinflammatory and antinociceptive effects, normalization of endogenous HA synthesis, and chondroprotection. ${ }^{13}$

PRP is a plasma fraction obtained by means of different methods of centrifugation, with platelet counts above the normal blood values. The basic principle underlying the PRP therapeutic activity is to deliver a large pool of signaling proteins, such as growth factors (GFs) and other cytokines, including platelet-derived growth factor (PDGF), transforming growth factor-1- $\beta$ (TGF1- $\beta$ ), vascular endothelial growth factor (VEGF), hepatocyte growth factor (HGF), interleukin-8
(IL-8), chemokine ligand 12 (CXCL12), chemokines, angiopoietin, and matrix metalloproteinases (MMPs) that drive tissue regeneration mechanisms. These regulatory proteins may be capable of interfering with various healing mechanisms that influence the inflammatory response, induce cell migration, proliferation, and modulate angiogenesis. ${ }^{14,15}$

The literature describes PRP as a new treatment for joint degenerative pathologies, and an increasing number of studies show promising results. ${ }^{16-18}$ Despite its wide clinical application, only a few reports have documented results for PRP in the treatment of hip degenerative lesions in the literature. ${ }^{4-8}$

The results of this trial suggested a possible effect of platelet concentrate on the treatment of hip OA, with a clinical subjective improvement but not significantly better results with respect to HA.

The results of this study also underlined some important aspects related to adequacy and safety of treatment. PRP did not offer better results compared with HA in this series. However, it has to be emphasized that the average age of the enrolled patients was higher than those of other studies, which reported a worse outcome for older patients treated with PRP. ${ }^{16,17}$

The safety of this procedure was confirmed. A higher pain reaction after PRP injection was found, probably due to the leukocyte content of our platelet concentrate, but without jeopardizing clinical results up to 1-year follow-up.

Cellularity is one of the most debated aspects when evaluating PRP properties and the results obtained with its application. In fact, not only platelets but also leukocytes, monocytes, macrophages, and mast cells are present in many platelet concentrates. Some authors define PRP as only platelets and attribute better results to leucocyte depletion, because of the deleterious effects of proteases and reactive oxygen species released from white cells; others consider them as a source of important cytokines and enzymes. ${ }^{19-23}$

In conclusion, PRP remains a debated treatment for hip OA. Despite its wide application in clinical practice and the positive findings reported, there is a lack of scientific background to guide the clinical application of PRP. To avoid indiscriminate and inappropriate use of PRP, it is important to determine the type of patients who will not benefit from this treatment. The results of this study suggest that for middle-aged patients with moderate signs of OA, PRP did not offer better results compared with HA, and thus it should not be considered as the first-line treatment. 


\section{References}

1 Robinson P, Keenan AM, Conaghan PG. Clinical effectiveness and dose response of image-guided intra-articular corticosteroid injection for hip osteoarthritis. Rheumatology (Oxford) 2007;46(02):285-291

2 Abate M, Pelotti P, De Amicis D, Di Iorio A, Galletti S, Salini V. Viscosupplementation with hyaluronic acid in hip osteoarthritis (a review). Ups J Med Sci 2008;113(03):261-277

3 Richette P, Ravaud P, Conrozier T, et al. Effect of hyaluronic acid in symptomatic hip osteoarthritis: a multicenter, randomized, placebo-controlled trial. Arthritis Rheum 2009;60(03):824-830

4 Battaglia M, Guaraldi F, Vannini F, et al. Platelet-rich plasma (PRP) intra-articular ultrasound-guided injections as a possible treatment for hip osteoarthritis: a pilot study. Clin Exp Rheumatol 2011;29(04):754

5 Sánchez M, Guadilla J, Fiz N, Andia I. Ultrasound-guided plateletrich plasma injections for the treatment of osteoarthritis of the hip. Rheumatology (Oxford) 2012;51(01):144-150

6 Battaglia M, Guaraldi F, Vannini F, et al. Efficacy of ultrasound-guided intra-articular injections of platelet-rich plasma versus hyaluronic acid for hip osteoarthritis. Orthopedics 2013;36(12):e1501-e1508

7 Di Sante L, Villani C, Santilli V, et al. Intra-articular hyaluronic acid vs platelet-rich plasma in the treatment of hip osteoarthritis. Med Ultrason 2016;18(04):463-468

8 Bennell KL, Hunter DJ, Paterson KL. Platelet-rich plasma for the management of hip and knee osteoarthritis. Curr Rheumatol Rep 2017;19(05):24

9 Meloni F, Milia F, Cavazzuti M, et al. Clinical evaluation of sodium hyaluronate in the treatment of patients with sopraspinatus tendinosis under echographic guide: experimental study of periarticular injections. Eur J Radiol 2008;68(01):170-173

10 Atchia I, Kane D, Reed MR, Isaacs JD, Birrell F. Efficacy of a single ultrasound-guided injection for the treatment of hip osteoarthritis. Ann Rheum Dis 2011;70(01):110-116

11 Mei-Dan O, McConkey MO, Petersen B, McCarty E, Moreira B, Young DA. The anterior approach for a non-image-guided intraarticular hip injection. Arthroscopy 2013;29(06):1025-1033

12 Salaffi F, Leardini G, Canesi B, et al; GOnorthrosis and Quality of Life Assessment (GOQOLA). Reliability and validity of the Western Ontario and McMaster Universities (WOMAC) Osteoarthritis Index in Italian patients with osteoarthritis of the knee. Osteoarthritis Cartilage 2003;11(08):551-560
13 Dettoni F, Pellegrino P, La Russa MR, et al. Validation and cross cultural adaptation of the Italian version of the Harris Hip Score. Hip Int 2015;25(01):91-97

14 Migliore A, Procopio S. Effectiveness and utility of hyaluronic acid in osteoarthritis. Clin Cases Miner Bone Metab 2015;12(01): 31-33

15 Anitua E, Sánchez M, Nurden AT, et al. Platelet-released growth factors enhance the secretion of hyaluronic acid and induce hepatocyte growth factor production by synovial fibroblasts from arthritic patients. Rheumatology (Oxford) 2007;46(12): 1769-1772

16 Caggiari G, Mosele GR, Puddu L, Doria C. Efficacy of platelet-rich plasma in experimental instrumented interbody spinal fusion. EuroMediterranean Biomed J 2016;11(10):141-147

17 Anitua E, Sánchez M, Zalduendo MM, et al. Fibroblastic response to treatment with different preparations rich in growth factors. Cell Prolif 2009;42(02):162-170

18 Kon E, Buda R, Filardo G, et al. Platelet-rich plasma: intra-articular knee injections produced favorable results on degenerative cartilage lesions. Knee Surg Sports Traumatol Arthrosc 2010;18(04): 472-479

19 Filardo G, Kon E, Buda R, et al. Platelet-rich plasma intra-articular knee injections for the treatment of degenerative cartilage lesions and osteoarthritis. Knee Surg Sports Traumatol Arthrosc 2011;19 (04):528-535

20 Peerbooms JC, Sluimer J, Bruijn DJ, Gosens T. Positive effect of an autologous platelet concentrate in lateral epicondylitis in a double-blind randomized controlled trial: platelet-rich plasma versus corticosteroid injection with a 1-year follow-up. Am J Sports Med 2010;38(02):255-262

21 Gosens T, Peerbooms JC, van Laar W, den Oudsten BL. Ongoing positive effect of platelet-rich plasma versus corticosteroid injection in lateral epicondylitis: a double-blind randomized controlled trial with 2-year follow-up. Am J Sports Med 2011;39 (06):1200-1208

22 de Vos RJ, Weir A, van Schie HTM, et al. Platelet-rich plasma injection for chronic Achilles tendinopathy: a randomized controlled trial. JAMA 2010;303(02):144-149

23 Mei-Dan O, Lippi G, Sánchez M, Andia I, Maffulli N. Autologous platelet-rich plasma: a revolution in soft tissue sports injury management? Phys Sportsmed 2010;38(04):127-135 